\title{
Liquid Phase Electron Microscopy Study on the Growth Mechanism of Gold Nanoparticles: In Scanning Electron Microscope
}

\author{
In Hyang Kang ${ }^{1}$, Moon Deock Kim ${ }^{1}$ and Young Heon $\mathrm{KIM}^{2 *}$ \\ 1. Department of Physics, 99 Daehak-ro, Yuseong-gu, Daejeon, South Korea. \\ 2. Graduate School of Analytical Science and Technology, 99 Daehak-ro, Yuseong-gu, Daejeon, South \\ Korea. \\ * Corresponding author: y.h.kim@cnu.ac.kr
}

Liquid phase electron microscopy is a novel method to observe materials in various liquids with high spatial resolution and real-time observation, which is implemented through a liquid cell in TEM [1-3]. This new technology is widely used to a variety of field, such as nanoparticle synthesis in liquids, the reaction in battery science, living cell study, and electrochemical researches. In addition, recently, many studies have been conducted to investigate the possibility of a direct synthesis, manipulation, and/or elimination of materials in liquids by using electron beam at the nanometer scale. For these studies, the studies on the reaction mechanisms between incident electrons and reacting materials, including the environmental liquids, are essential required. Specifically, a scanning electron microscope (SEM) is the standout instrument to use electron beam for preparing and manipulating targeted nanomaterials in liquids because it is possible to easily control the boundary conditions and it has the wide available space to utilize in the instrument.

The formation of gold (Au) nanoparticles (NPs) by the incident electrons have been studied in SEM, based on the development of the small devices for seeking liquid environments. The growth of Au NPs was monitored in real time. The microstructural properties of Au NPs were studied by analyzing the atomic structures. The interactions between incident electrons accelerated at low voltages and liquids are discussed and the experimental results are demonstrated based on the theoretical consideration. Specially, the inelastic scattering of the incident electrons were deeply considered on the precursor materials because the reactant atoms can be generated by the phenomenon. Although several research papers have reported the growth behaviors of Au NPs, the understanding on the growth mechanism is insufficient to control the physical and chemical properties [4-6].

The in situ observation shows that area growing rate of Au NPs depends on time and the analysis result indicates that $\mathrm{Au}$ NPs were grown by following the reaction limited growth model. Different shapes and/or morphologies of Au NPs were observed at the initial stage of the growth: circular shape and triangular plate. The circular Au NPs kept their morphological features during the growth. However, the triangular plate of $\mathrm{Au} \mathrm{NPs}$ at the initial stage were propagated to two different shape; triangular and hexagonal shapes (Figure 1). And three-dimensional (3D) shapes of Au NPs were demonstrated by considering the signal intensities for various 2 dimensional (2D) images and by gathering secondary electrons from the NPs. Many faulted regions, including twin boundaries, were observed in the circular Au NPs while the triangular and/or hexagonal plates were free from any crystalline defects. 


\section{References:}

[1] N de Jonge and F Ross, Nat. Nanotech. 6 (2011), p. 695.

[2] F Ross, Science 350 (2015), p. 1490.

[3] M Williamson et al., Nat. Mater. 2 (2003), p. 532.

[4] J Evans et al., Nano Lett. 11 (2011), p. 2809.

[5] C Zhu et al., Nat. Comm. 9 (2018), p. 421.

[6] C Li et al., Nano Res. 11 (2018), p. 4697.
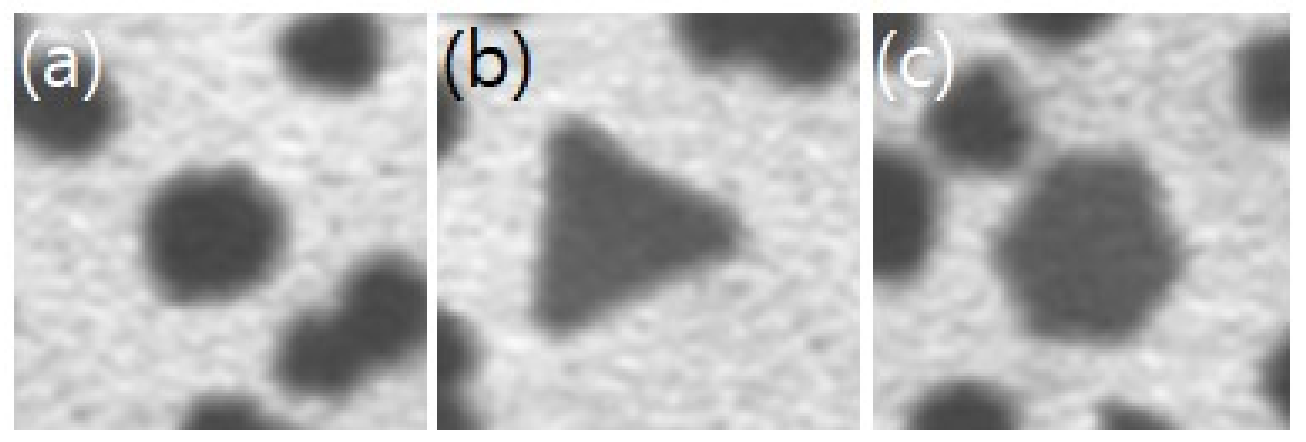

Figure 1. STEM images of Au NPs taken at the same time in SEM. (a) Circular shape, (b) Triangular plate, (c) Hexagonal plate. 